\title{
An $M^{[X]} / G / 1$ Queue with Bernoulli Schedule General Vacation Times, General Extended Vacations, Random Breakdowns, General Delay Times for Repairs to Start and General Repair Times
}

\author{
Rehab F. Khalaf (Correponding author) \\ School of Information Systems Computing and Mathematics \\ Brunel University, UK \\ Tel: 44-965-6601-3912 E-mail: Rehab.Khalaf@brunel.ac.uk, rihabfadil@gmail.com \\ Kailash C. Madan \\ Ahlia University, College of Information Technology, Kingdom of Bahrain \\ E-mail: kailash@ahliauniversity.edu.bh,kcmadan@yahoo.com \\ Cormac A. Lukas \\ School of Information Systems Computing and Mathematics \\ Brunel University, UK \\ E-mail: cormac.lucas@brunel.ac.uk
}

Received: July 7, 2011 Accepted: July 18, 2011 Published: November 1, 2011

doi:10.5539/jmr.v3n4p8 URL: http://dx.doi.org/10.5539/jmr.v3n4p8

\begin{abstract}
This paper studies a batch arrival queueing system in which the server may face occasional random breakdowns. The repair process does not start immediately after a breakdown and there is a delay time waiting for repairs to start. Further, after every service completion the server has the option to leave for a vacation with probability $p$ or continue serving with probability $1-p$. The main new assumption in this paper is that the server has the option to go on extended vacation after the original vacation completion with probability $r$ or rejoins the system to provide service directly after the original vacation with probability $1-r$. The service times, vacation times, extended vacation times, delay times and repair times are all assumed to follow general arbitrary distributions, while only the breakdown times are exponentially distributed.
\end{abstract}

Keywords: $M^{[X]} / G / 1$ queue, Batch arrival, Vacation time, Extended vacation time, Random breakdown, Delay time, Repair time

\section{Introduction}

Several authors including (Y. Levy, U. Yechilai,1976), (Doshi, B. T., 1986), (Takagi, H., 1990) and (Madan, 1991, 2000b, 2005) have studied queues with vacations. Also queueing systems with breakdowns have been studied by several authors including (Federgruen \& So, 1990), (Aissani \& Artalejo, 1998), (Wang, Cao \& Li, 2001) and (Madan, Abu-Dayyeh, Gharaibeh, 2003b). Recently (Maraghi, Madan and Darby-Dowman, 2009 a) have studied some queueing systems with vacations and breakdowns. All these research papers assume no server delay in joining the system after completion of a vacation period and no delay in starting the repair process after a breakdown occurs. In this work we study an $M^{[X]} / G / 1$ queueing system with Bernoulli schedule vacation where after completion of the service of a customer the server may take a vacation with probability $p$ or stay on in the system to serve the customers with probability $1-p$ In most of the studies on vacation queues, it is assumed that the server joins the queue immediately after the vacation period is over. However, in this paper we add a realistic assumption that the server may have to go for an extended vacation due to many reasons which may include illness or engagement in another activity or delay in traveling etc.

We further assume that the server may face random breakdowns from time to time. When the server breaks down, it does not enter the repair process immediately and there is a delay time waiting for the repair to start.

We assume that the service times, vacation times, extended vacation times, repair times and delay times have a general 
distribution while the breakdown times are exponentially distributed.

The rest of this work is organized as follows: Section 2 gives the mathematical model containing the assumptions underlying the considered queueing system. Related definitions and used notation are given in Section 3. Equations governing the system are formulated in Section 4. In section 5 we give the solution of the equations given in the previous section to find the queue size distribution at a random epoch. The average queue size and the average waiting time are given in Section 6. Some special cases have been discussed in Section 7. In Section 8, we consider a numerical example to illustrate application of our results.

\section{Mathematical Model}

We consider a batch arrival queueing system, where customers arrive at the system according to a compound Poisson process with batch size random variable $X$ and batch arrival rate $\lambda$. Let $c_{k}=\operatorname{Pr}[X=k]$, then $\lambda c_{k} d t$ is the probability that during a short time interval $(t, t+\Delta t)$ a batch of size $k(k=1,2,3, \ldots)$ arrives the system.

Although the customers arrive in groups, the single server can serve only one customer at a time based on the (FCFS) discipline. The service times assumed to follow a general distribution. Let $G(x)$ and $g(x)$ be the distribution function and the density function of the service time respectively. The conditional probability of service completion during the interval, $(x, x+\Delta x]$, given that the elapsed service time is $x$, is given by $\mu(x) \Delta x$, so that $\mu(x)=g(x) /(1-G(x))$ and, therefore $g(s)=\mu(s) \exp \left(-\int_{0}^{s} \mu(x) d x\right)$. Once the single server complete a customer service it can go on a vacation of random length with probability $p$, or stay in the system providing service with probability $1-p$. The vacation times are assumed to follow a general distribution. Let $B(x)$ and $b(x)$ be the distribution function and the density function of the vacation time respectively.The conditional probability of a vacation completion during the interval, $(x, x+\Delta x]$,given that the elapsed vacation time is $x$, is given by $\beta(x) \Delta x$, so that $\beta(x)=b(x) /(1-B(x))$ implies to $b(v)=\beta(v) \exp \left(-\int_{0}^{v} \beta(x) d x\right)$. On completion of a vacation period, the server has the further option of taking an extended vacation. We assume that with probability $r(0 \leq r \leq 1)$ the server takes an extended vacation and with probability $1-$ rrejoins the system immediately after completion of the first vacation. The extended vacation times follows a general (arbitrary) distribution with distribution function $W(x)$ and density function $w(x)$. Let $\varphi(x) \Delta x$ be the conditional probability of a completion of an extended vacation during the interval $(x, x+\Delta x]$ given that the elapsed extended vacation time is $x$, so that $\varphi(x)=w(x) /(1-W(x))$ and therefore, $w(x)=\varphi(x) \exp \left(-\int_{0}^{x} \varphi(t) d t\right)$. The system may breakdown at random, and breakdowns are assumed to occur according to a Poisson stream with mean breakdown rate $\alpha>0$. Further we assume that once the system breaks down, the customer whose service is interrupted comes back to the head of the queue. Once the system breakdown, its repairs do not start immediately and there is a delay time.

The delay times follow a general (arbitrary) distribution with distribution function $F(x)$ and density function $f(x)$. Let $\theta(x) \Delta x$ be the conditional probability of a completion of a delay during the interval $(x, x+\Delta x]$ given that the elapsed delay time is $x$, so that, $\theta(x)=f(x) /(1-F(x))$ and therefore, $f(x)=\theta(x) \exp \left(-\int_{0}^{x} \theta(t) d t\right)$.

The duration of repairs follows a general (arbitrary) distribution with distribution function $H(x)$ and density function $h(x)$. Let $\gamma(x) \Delta x$ be the conditional probability of a completion of repairs during the interval $(x, x+\Delta x]$ given that the elapsed repair time is $x$, so that $\gamma(x)=h(x) /(1-H(x))$ and therefore $h(x)=\gamma(x) \exp \left(-\int_{0}^{x} \gamma(t) d t\right)$. We assume that all stochastic processes involved in the system are independent of each other.

\section{Definitions and notations}

The set of notations and their definitions that we use are set out in table 1.

Assuming that the steady state exists, we let

$$
\begin{aligned}
& \lim _{t \rightarrow \infty} A_{n}(t, x)=A_{n}(x), \quad \lim _{t \rightarrow \infty} A_{n}(t)=\lim _{t \rightarrow \infty} \int_{0}^{\infty} A_{n}(t, x) d x=A_{n}, \\
& \lim _{t \rightarrow \infty} \frac{d A_{n}(t)}{d t}=0, \quad \text { where } A=P, V, D, E, R
\end{aligned}
$$

\section{Equations Governing the System}

According to the assumptions mentioned above, the system has the following set of differential-difference equations 


$$
\begin{aligned}
& \frac{\partial}{\partial x} P_{n}(x)=-(\lambda+\mu(x)+\alpha) P_{n}(x)+\lambda \sum_{i=1}^{n} c_{i} P_{n-i}(x) \quad n \geq 1 \\
& \frac{\partial}{\partial x} P_{0}(x)=-(\lambda+\mu(x)+\alpha) P_{0}(x) \\
& \frac{\partial}{\partial x} V_{n}(x)=-(\lambda+\beta(x)) V_{n}(x)+\lambda \sum_{i=1}^{n} c_{i} V_{n-i}(x) \quad n \geq 1 \\
& \frac{\partial}{\partial x} V_{0}(x)=-(\lambda+\beta(x)) V_{0}(x) \\
& \frac{\partial}{\partial x} E_{n}(x)+(\lambda+\varphi(x)) E_{n}(x)=\lambda \sum_{i=1}^{n} c_{i} E_{n-i}(x) \quad n \geq 1 \\
& \frac{\partial}{\partial x} E_{0}(x)=-(\lambda+\varphi(x)) E_{0}(x) \\
& \frac{\partial}{\partial x} D_{n}(x)+(\lambda+\theta(x)) D_{n}(x)=\lambda \sum_{i=1}^{n} c_{i} D_{n-i}(x) \quad n \geq 1 \\
& \frac{\partial}{\partial x} D_{0}(x)=0 \\
& \frac{\partial}{\partial x} R_{n}(x)+(\lambda+\gamma(x)) R_{n}(x)=\lambda \sum_{i=1}^{n} c_{i} R_{n-i}(x) \quad n \geq 1 \\
& \frac{\partial}{\partial x} R_{0}(x)=-(\lambda+\gamma(x)) R_{0}(x)=0 \\
& \lambda Q=\int_{0}^{\infty} R_{0}(x) \gamma(x) d x+(1-p) \int_{0}^{\infty} P_{0}(x) \mu(x) d x+(1-r) \int_{0}^{\infty} V_{0}(x) \beta(x) d x+\int_{0}^{\infty} E_{0}(x) \varphi(x) d x
\end{aligned}
$$

The following boundary conditions will be used to solve the above equations

$$
\begin{gathered}
P_{n}(0)=(1-p) \int_{0}^{\infty} P_{n+1}(x) \mu(x) d x+(1-r) \int_{0}^{\infty} V_{n+1}(x) \beta(x) d x+\int_{0}^{\infty} E_{n+1}(x) \varphi(x) d x \\
+\int_{0}^{\infty} R_{n+1}(x) \gamma(x) d x+\lambda c_{n+1} Q \quad n \geq 0 \\
V_{n}(0)=p \int_{0}^{\infty} P_{n}(x) \mu(x) d x \quad n \geq 0 \\
E_{n}(0)=r \int_{0}^{\infty} V_{n}(x) \beta(x) d x \quad n \geq 0 \\
D_{n}(0)=\alpha \int_{0}^{\infty} P_{n-1}(x) d x=\alpha P_{n-1} \quad n \geq 1
\end{gathered}
$$




$$
\begin{gathered}
D_{0}(0)=0 \\
R_{n}(0)=\int_{0}^{\infty} D_{n}(x) \theta(x) d x \quad n \geq 1 \\
R_{0}(0)=0
\end{gathered}
$$

\section{Queue Size Distribution at a Random Epoch}

Defining the following probability generating functions

$$
\begin{aligned}
& A_{q}(x, z)=\sum_{n=0}^{\infty} z^{n} A_{n}(x) \quad A_{q}(z)=\sum_{n=0}^{\infty} z^{n} A_{n} \\
& \text { where } A=P, V, D, E, R \\
& C(z)=\sum_{i=1}^{\infty} z^{i} c_{i}
\end{aligned}
$$

We Multiply equation (2) by $z^{n}$, take the summation over $\mathrm{n}$ from 1 to $\infty$, add to (3) then by simplifying and using equation (20) to obtain

$$
\frac{\partial}{\partial x} P_{q}(x, z)+(\lambda-\lambda C(z)+\mu(x)+\alpha) P_{q}(x, z)=0
$$

Following a similar process, from equations (4) \& (5), (6) \& (7), (8) \& (9), (10) \& (11). we get respectively

$$
\begin{aligned}
& \frac{\partial}{\partial x} V_{q}(x, z)+(\lambda-\lambda C(z)+\beta(x)) V_{q}(x, z)=0 \\
& \frac{\partial}{\partial x} E_{q}(x, z)+(\lambda-\lambda C(z)+\varphi(x)) E_{q}(x, z)=0 \\
& \frac{\partial}{\partial x} D_{q}(x, z)+(\lambda-\lambda C(z)+\theta(x)) D_{q}(x, z)=0 \\
& \frac{\partial}{\partial x} R_{q}(x, z)+(\lambda-\lambda C(z)+\gamma(x)) R_{q}(x, z)=0
\end{aligned}
$$

Multiply equation (13) by $z^{n+1}$, sum over $n$ from 0 to $\infty$, and use the generating functions defined in (20), we get

$$
\begin{array}{r}
z P_{q}(0, z)=(1-p) \int_{0}^{\infty} P_{q}(x, z) \mu(x) d x+(1-r) \int_{0}^{\infty} V_{q}(x, z) \beta(x) d x+\int_{0}^{\infty} E_{q}(x, z) \varphi(x) d x+\int_{0}^{\infty} R_{q}(x, z) \gamma(x) d x+\lambda C(z) Q \\
-\left[(1-p) \int_{0}^{\infty} P_{0}(x) \mu(x) d x+(1-r) \int_{0}^{\infty} V_{0}(x, z) \beta(x) d x+\int_{0}^{\infty} E_{0}(x) \varphi(x) d x+\int_{0}^{\infty} R_{0}(x) \gamma(x) d x\right]
\end{array}
$$

using equation (12) to Replace $-\left[(1-p) \int_{0}^{\infty} P_{0}(x) \mu(x) d x+(1-r) \int_{0}^{\infty} V_{0}(x, z) \beta(x) d x+\int_{0}^{\infty} E_{0}(x) \varphi(x) d x+\int_{0}^{\infty} R_{0}(x) \gamma(x) d x\right]$ by $-\lambda Q$, we have

$$
\begin{aligned}
z P_{q}(0, z) & =(1-p) \int_{0}^{\infty} P_{q}(x, z) \mu(x) d x+\int_{0}^{\infty} E_{q}(x, z) \varphi(x) d x+(1-r) \int_{0}^{\infty} V_{q}(x, z) \beta(x) d x \\
+ & \int_{0}^{\infty} R_{q}(x, z) \gamma(x) d x+\lambda Q(C(z)-1)
\end{aligned}
$$


Now multiply equation (14) by $z^{n}$ and sum over $n$ from 0 to $\infty$, to obtain

$$
V_{q}(0, z)=p \int_{0}^{\infty} P_{q}(x, z) \mu(x) d x
$$

Similarly, we multiply equation (15) by $z^{n}$ and sum over $n$ from 0 to $\infty$, we get

$$
E_{q}(0, z)=r \int_{0}^{\infty} V_{q}(x, z) \beta(x) d x
$$

Multiply equation (16) by $z^{n}$ and sum over $n$ from 1 to $\infty$ and taking into account (17), we get

$$
D_{q}(0, z)=\alpha z P_{q}(z)
$$

Now multiply equation (18) by $z^{n}$ and sum over $n$ from 1 to $\infty$ and taking into account (19), we get

$$
R_{q}(0, z)=\int_{0}^{\infty} D_{q}(x, z) \theta(x) d x
$$

Integrating equation (21) from 0 to $x$ yields

$$
P_{q}(x, z)=P_{q}(0, z) e^{-(\lambda-\lambda C(z)+\alpha) x-\int_{0}^{x} \mu(t) d t}
$$

where $P_{q}(0, z)$ is given by equation (57).

Let $\lambda-\lambda C(z)+\alpha=a$ Integrating equation (32) by parts with respect to $x$ yields

$$
P_{q}(z)=P_{q}(0, z)\left(\frac{1-G^{*}(a)}{a}\right)
$$

where $G^{*}(a)=\int_{0}^{\infty} e^{-(\lambda-\lambda C(z)+\alpha) x} d G(x)$ is the Laplace-Stieltjes transform of the service time $G(x)$

Now multiplying both sides of equation (32) by $\mu(x)$ and integrating over $x$, we get

$$
\int_{0}^{\infty} P_{q}(x, z) \mu(x) d x=P_{q}(0, z) G^{*}(a)
$$

Using equation (34) from equation (28), we get

$$
V_{q}(0, z)=p P_{q}(0, z) G^{*}(a)
$$

Similarly, we integrate equation (22) from 0 to $x$, we get

$$
V_{q}(x, z)=V_{q}(0, z) e^{-(\lambda-\lambda C(z)) x-\int_{0}^{x} \beta(t) d t}
$$

Substituting by the value of $V_{q}(0, z)$ from (35) in equation (36), we get

$$
V_{q}(x, z)=p P_{q}(0, z) G^{*}(a) e^{-(\lambda-\lambda C(z)) x-\int_{0}^{x} \beta(t) d t}
$$

Let $\lambda-\lambda C(z)=b$. Integrating equation (37) by parts with respect to $x$, we get 


$$
V_{q}(z)=\frac{p P_{q}(0, z) G^{*}(a)\left(1-B^{*}(b)\right)}{b}
$$

where $B^{*}(b)=\int_{0}^{\infty} e^{-(\lambda-\lambda C(z)) x} d B(x)$ is the Laplace-Stieltjes transform of the vacation time $B(x)$.

Now multiplying both sides of equation (37) by $\beta(x)$ and integrating over $x$, we get

$$
\int_{0}^{\infty} V_{q}(x, z) \beta(x) d x=p P_{q}(0, z) G^{*}(a) B^{*}(b)
$$

Integrating equation (23) from 0 to $x$ and taking into account equations (29) and (39). we get

$$
E_{q}(x, z)=E_{q}(0, z) e^{-b x-\int_{0}^{x} \varphi(t) d t}=r p P_{q}(0, z) G^{*}(a) B^{*}(b) e^{-b x-\int_{0}^{x} \varphi(t) d t}
$$

By integrating equation (40) by parts with respect to $x$, we get

$$
E_{q}(z)=\frac{r p P_{q}(0, z) G^{*}(a) B^{*}(b)\left(1-W^{*}(b)\right)}{b}
$$

where $W^{*}(b)=\int_{0}^{\infty} e^{-(\lambda-\lambda C(z)) x} d W(x)$ is the Laplace-Stieltjes transform of the extended vacation time $W(x)$.

Now multiplying both sides of equation (40) by $\varphi(x)$ and integrating over $x$, we get

$$
\int_{0}^{\infty} E_{q}(x, z) \varphi(x) d x=r p P_{q}(0, z) G^{*}(a) B^{*}(b) W^{*}(b)
$$

Integrating equation (24) from 0 to $x$ and taking into account the value of $D_{q}(0, z)$ from (30), we get

$$
D_{q}(x, z)=D_{q}(0, z) e^{-b x-\int_{0}^{x} \theta(t) d t}=\alpha z P_{q}(z) e^{-b x-\int_{0}^{x} \theta(t) d t}
$$

Integrating equation (43) by parts with respect to $x$ and taking into account equation (33), we get

$$
D_{q}(z)=\frac{\alpha z P_{q}(z)\left(1-F^{*}(b)\right)}{b}=\frac{\alpha z P_{q}(0, z)\left(1-G^{*}(a)\right)\left(1-F^{*}(b)\right)}{a b}
$$

where $F^{*}(b)=\int_{0}^{\infty} e^{-(\lambda-\lambda C(z)) x} d F(x)$ is the Laplace-Stieltjes transform of the delay time $F(x)$.

Now multiplying both sides of equation (43) by $\theta(x)$, integrating over $x$ and taking into account equation (33), we get

$$
\int_{0}^{\infty} D_{q}(x, z) \theta(x) d x=\alpha z P_{q}(z) F^{*}(b)=\frac{\alpha z P_{q}(0, z)\left(1-G^{*}(a)\right) F^{*}(b)}{a}
$$

Now integrating equation (25) from 0 to $x$, and taking into account equations (31) and (45), we get

$$
R_{q}(x, z)=R_{q}(0, z) e^{-b x-\int_{0}^{x} \gamma(t) d t}=\frac{\alpha z P_{q}(0, z)\left(1-G^{*}(a)\right) F^{*}(b)}{a} e^{-b x-\int_{0}^{x} \gamma(t) d t}
$$

integrating equation (46) by parts with respect to $x$, we get 


$$
R_{q}(z)=\frac{\alpha z P_{q}(0, z)\left(1-G^{*}(a)\right) F^{*}(b)\left(1-H^{*}(b)\right)}{a b}
$$

where $H^{*}(b)=\int_{0}^{\infty} e^{-(\lambda-\lambda C(z)) x} d H(x)$ is the Laplace-Stieltjes transform of the repair time $H(x)$.

Multiplying both sides of equation (46) by $\gamma(x)$ and integrating over $x$, we get

$$
\int_{0}^{\infty} R_{q}(x, z) \gamma(x) d x=\frac{\alpha z P_{q}(0, z)\left(1-G^{*}(a)\right) F^{*}(b) H^{*}(b)}{a}
$$

Using equations (34), (39), (42) and (48) in equation (27) yields

$$
P_{q}(0, z)=\frac{-a b Q}{a\left(z-G^{*}(a)\left(1-p+p B^{*}(b)\left(1-r+r W^{*}(b)\right)\right)\right)-\alpha z\left(1-G^{*}(a)\right) F^{*}(b) H^{*}(b)}
$$

Substituting $P_{q}(0, z)$ in equation (33), (38), (41), (44) and (47), we obtain

$$
\begin{aligned}
& P_{q}(z)=\frac{-b Q\left(1-G^{*}(a)\right)}{a\left(z-G^{*}(a)\left(1-p+p B^{*}(b)\left(1-r+r W^{*}(b)\right)\right)\right)-\alpha z\left(1-G^{*}(a)\right) F^{*}(b) H^{*}(b)} \\
& V_{q}(z)=\frac{-a Q p G^{*}(a)\left(1-B^{*}(b)\right)}{a\left(z-G^{*}(a)\left(1-p+p B^{*}(b)\left(1-r+r W^{*}(b)\right)\right)\right)-\alpha z\left(1-G^{*}(a)\right) F^{*}(b) H^{*}(b)} \\
& E_{q}(z)=\frac{-a Q r p G^{*}(a) B^{*}(b)\left(1-W^{*}(b)\right)}{a\left(z-G^{*}(a)\left(1-p+p B^{*}(b)\left(1-r+r W^{*}(b)\right)\right)\right)-\alpha z\left(1-G^{*}(a)\right) F^{*}(b) H^{*}(b)} \\
& D_{q}(z)=\frac{-Q \alpha z\left(1-G^{*}(a)\right)\left(1-F^{*}(b)\right)}{a\left(z-G^{*}(a)\left(1-p+p B^{*}(b)\left(1-r+r W^{*}(b)\right)\right)\right)-\alpha z\left(1-G^{*}(a)\right) F^{*}(b) H^{*}(b)} \\
& R_{q}(z)=\frac{-Q \alpha z\left(1-G^{*}(a)\right) F^{*}(b)\left(1-H^{*}(b)\right)}{a\left(z-G^{*}(a)\left(1-p+p B^{*}(b)\left(1-r+r W^{*}(b)\right)\right)\right)-\alpha z\left(1-G^{*}(a)\right) F^{*}(b) H^{*}(b)}
\end{aligned}
$$

Let $S_{q}(z)$ denote the probability generating function of the queue size irrespective of the state of the system. i.e $S_{q}(z)=$ $P_{q}(z)+V_{q}(z)+E_{q}(z)+D_{q}(z)+R_{q}(z)$.

Then adding equations (50), (51), (52), (53) and (54), we obtain

$$
S_{q}(z)=\frac{-Q\left(1-G^{*}(a)\right)\left\{b+\alpha z\left(1-F^{*}(b) H^{*}(b)\right)\right\}-a Q p G^{*}(a)\left\{1-B^{*}(b)\left(1-r+r W^{*}(b)\right)\right\}}{a\left(z-G^{*}(a)\left(1-p+p B^{*}(b)\left(1-r+r W^{*}(b)\right)\right)\right)-\alpha z\left(1-G^{*}(a)\right) F^{*}(b) H^{*}(b)}
$$

In order to find $Q$, we use the normalization condition $S_{q}(1)+Q=1$.

Note that for $z=1, S_{q}(1)$ is indeterminate of the $0 / 0$ form. Therefore, we apply L'Hopitals Rule on equation (55), to obtain

$$
\lim _{z \rightarrow 1} S_{q}(z)=\frac{Q \lambda E(I)\left\{\left(1-G^{*}(\alpha)\right)\left(1+\alpha(E(D)+E(R))+\alpha p G^{*}(\alpha)(E(V)+r E(e V))\right\}\right.}{-\alpha p \lambda E(I) G^{*}(\alpha)(E(V)+r E(e V))-\lambda E(I)\left(1-G^{*}(a)\right)[1+\alpha(E(D)+E(R))]+\alpha G^{*}(\alpha)}
$$

where $C(1)=1, C^{\prime}(1)=E(I)$ is the mean batch size of the arriving customers, $B *(0)=1$ and $B *^{\prime}(0)=-E(V)$ the mean vacation time, $W *(0)=1$ and $W *^{\prime}(0)=-E(\mathrm{eV})$ the mean extended vacation time, $F *(0)=1$, and $F *^{\prime}(0)=-E(D)$ the mean delay time and $H *(0)=1$, and $H *^{\prime}(0)=-E(R)$ the mean repair time.

Therefore, adding $Q$ to equation (61) and equating to 1 and simplifying, we get

$$
Q=1-\lambda E(I)\left[\frac{1}{\alpha G^{*}(\alpha)}+\frac{E(D)}{G^{*}(\alpha)}+\frac{E(R)}{G^{*}(\alpha)}-\frac{1}{\alpha}-E(D)-E(R)+p(E(V)+r E(e V))\right]
$$


Equation (57) gives the probability that the server is idle. From equation (57) the utilization factor, $\rho$ of the system is given by

$$
\rho=\lambda E(I)\left[\frac{1}{\alpha G^{*}(\alpha)}+\frac{E(D)}{G^{*}(\alpha)}+\frac{E(R)}{G^{*}(\alpha)}-\frac{1}{\alpha}-E(D)-E(R)+p(E(V)+r E(e V))\right]
$$

where $\rho<1$ is the stability condition under which the steady states exits.

Substituting for $Q$ from (57) into (55), we have completely and explicitly determined $S_{q}(z)$, the probability generating function of the queue size.

\section{The Average Queue Size and the Average Waiting Time}

Let $L_{q}$ denote the mean number of customers in the queue under the steady state. Then

$$
L_{q}=\left.\frac{d}{d z} S_{q}(z)\right|_{z=1}
$$

Since this formula is of the $0 / 0$ form, then we write $S_{q}(z)$ given in (55) as $S_{q}(z)=N(z) / D(z)$ where $N(z)$ and $D(z)$ are the numerator and denominator of the right hand side of (55) respectively. Then using the L'Hopital's rule twice we obtain

$$
L_{q}=\lim _{z \rightarrow 1} \frac{D^{\prime}(z) N^{\prime \prime}(z)-N^{\prime}(z) D^{\prime \prime}(z)}{2\left(D^{\prime}(z)\right)^{2}}
$$

where primes and double primes in (60) denote first and second derivatives at $z=1$, respectively. Carrying out the derivatives at $z=1$, we have

$$
\begin{aligned}
N^{\prime}(1)=Q \lambda E(I)\left\{\left(1-G^{*}(\alpha)\right)(1+\alpha(E(D)+E(R)))+\alpha p Q G^{*}(\alpha)(E(V)+r E(e V))\right\} \\
N^{\prime \prime}(1)=(\lambda E(I))^{2} Q\left\{\alpha\left(1-G^{*}(\alpha)\right)\left[E\left(D^{2}\right)+E\left(R^{2}\right)+2 E(D) E(R)\right]+2 G^{*^{\prime}}(\alpha)(1+\alpha(E(D)+E(R)))\right. \\
\left.+\alpha p G^{*}(\alpha)\left[E\left(V^{2}\right)+r E\left(e V^{2}\right)+2 r E(V) E(e V)\right]-2 p(E(V)+r E(e V))\left(\alpha G^{*^{\prime}}(\alpha)+G^{*}(\alpha)\right)\right\} \\
+\lambda E(I(I-1)) Q\left\{\left(1-G^{*}(\alpha)\right)(1+\alpha(E(D)+E(R)))+\alpha p G^{*}(\alpha)(E(V)+r E(e V))\right\} \\
+2 \alpha Q \lambda E(I)\left(1-G^{*}(\alpha)\right)(E(D)+E(R)) \\
D^{\prime}(1)=-\lambda E(I)\left\{\left(1-G^{*}(\alpha)\right)(1+\alpha(E(D)+E(R)))+\alpha p G^{*}(\alpha)(E(V)+r E(e V))\right\}+\alpha G^{*}(\alpha) \\
\\
D^{\prime \prime}(1)=-(\lambda E(I))^{2} G^{*}(\alpha)\left\{\alpha p\left(E\left(V^{2}\right)+r E\left(e V^{2}\right)+2 r E(V) E(e V)\right)-2 p(E(V)+r E(e V))\right. \\
\left.-\alpha\left(E\left(D^{2}\right)+E\left(R^{2}\right)+2 E(D) E(R)\right)\right\}-\alpha(\lambda E(I))^{2}\left(E\left(D^{2}\right)+E\left(R^{2}\right)+2 E(D) E(R)\right) \\
-\lambda E(I(I-1))\left\{\left(1-G^{*}(\alpha)\right)\left(1+\alpha(E(D)+E(R))+\alpha p G^{*}(\alpha)(E(V)+r E(e V))\right\}\right. \\
-2(\lambda E(I))^{2} G^{*^{\prime}}(\alpha)\{1+\alpha(E(D)+E(R))-\alpha p(E(V)+r E(e V))\} \\
-2 \lambda E(I)\left\{1+\alpha G^{*^{\prime}}(\alpha)+\alpha\left(1-G^{*}(\alpha)\right)(E(D)+E(R))\right\}
\end{aligned}
$$

where $E\left(V^{2}\right), E\left(R^{2}\right), E\left(D^{2}\right), E\left(e V^{2}\right)$ are the second moment of the; vacation time, repair time, delay time and the extended vacation time respectively, $E(I(I-1))$ is the second factorial moment of the batch size of arriving customers, and $Q$ has been found in (57).

The following cases have been derived as some interesting particular cases of our results obtained in the previous section 6.

\subsection{Case 1. no delay for repairs to start}

Once the system breakdown, if its repairs start immediately and there is no delay time we let $E(D)=0$ and $F^{*}(b)=1$ Using this in the main results of this paper, we get

$$
\begin{gathered}
S_{q}(z)=\frac{-Q\left(1-G^{*}(a)\right)\left\{b+\alpha z\left(1-H^{*}(b)\right)\right\}-a Q p G^{*}(a)\left\{1-B^{*}(b)\left(1-r+r W^{*}(b)\right)\right\}}{a\left(z-G^{*}(a)\left(1-p+p B^{*}(b)\left(1-r+r W^{*}(b)\right)\right)\right)-\alpha z\left(1-G^{*}(a)\right) H^{*}(b)} \\
Q=1-\lambda E(I)\left[\frac{1}{\alpha G^{*}(\alpha)}+\frac{E(R)}{G^{*}(\alpha)}-\frac{1}{\alpha}-E(R)+p(E(V)+r E(e V))\right]
\end{gathered}
$$




$$
\begin{gathered}
N^{\prime}(1)=Q \lambda E(I)\left\{\left(1-G^{*}(\alpha)\right)(1+\alpha E(R))+\alpha p G^{*}(\alpha)(E(V)+r E(e V))\right\} \\
N^{\prime \prime}(1)=(\lambda E(I))^{2} Q\left\{\alpha\left(1-G^{*}(\alpha)\right) E\left(R^{2}\right)+2 G^{*^{\prime}}(\alpha)(1+\alpha(E(D)+E(R)))\right. \\
+\alpha p G^{*}(\alpha)\left[E\left(V^{2}\right)+r E\left(e V^{2}\right)+2 r E(V) E(e V)\right] \\
\left.\quad-2 p(E(V)+r E(e V))\left(\alpha G^{*^{\prime}}(\alpha)+G^{*}(\alpha)\right)\right\}+2 \alpha Q \lambda E(I)\left(1-G^{*}(\alpha)\right) E(R) \\
+\lambda E(I(I-1)) Q\left\{\left(1-G^{*}(\alpha)\right)(1+\alpha E(R))+\alpha p G^{*}(\alpha)(E(V)+r E(e V))\right\} \\
D^{\prime}(1)=-\lambda E(I)\left\{\left(1-G^{*}(\alpha)\right)(1+\alpha E(R))+\alpha p G^{*}(\alpha)(E(V)+r E(e V))\right\}+\alpha G^{*}(\alpha) \\
D^{\prime \prime}(1)=-(\lambda E(I))^{2} G^{*}(\alpha)\left\{\alpha p\left(E\left(V^{2}\right)+r E\left(e V^{2}\right)+2 r E(V) E(e V)\right)-2 p(E(V)+r E(e V))\right. \\
\left.-\alpha E\left(R^{2}\right)\right\}-\alpha(\lambda E(I))^{2} E\left(R^{2}\right) \\
-\lambda E(I(I-1))\left\{\left(1-G^{*}(\alpha)\right)(1+\alpha E(R))+\alpha p G^{*}(\alpha)(E(V)+r E(e V))\right\} \\
-2(\lambda E(I))^{2} G^{*^{\prime}}(\alpha)\{1+\alpha E(R)-\alpha p(E(V)+r E(e V))\} \\
-2 \lambda E(I)\left\{1+\alpha G^{*^{\prime}}(\alpha)+\alpha\left(1-G^{*}(\alpha)\right) E(R)\right\}
\end{gathered}
$$

The results obtained in (65) to (70) agree with the result given by the authors in previous work. (communicated for publication)

\subsection{Case 2. no extended vacation time}

Once the vacation ends finish the server start the service immediately and there is no extended vacation time, we let $r=0$ and $W^{*}(b)=1$. Using this in the main results of this paper, we get

$$
\begin{aligned}
& S_{q}(z)=\frac{-Q\left(1-G^{*}(a)\right)\left\{b+\alpha z\left(1-F^{*}(b) H^{*}(b)\right)\right\}-a Q p G^{*}(a)\left\{1-B^{*}(b)\right\}}{a\left(z-G^{*}(a)\left(1-p+p B^{*}(b)\right)\right)-\alpha z\left(1-G^{*}(a)\right) F^{*}(b) H^{*}(b)} \\
& Q=1-\lambda E(I)\left[\frac{1}{\alpha G^{*}(\alpha)}+\frac{E(D)}{G^{*}(\alpha)}+\frac{E(R)}{G^{*}(\alpha)}-\frac{1}{\alpha}-E(D)-E(R)+p E(V)\right] \\
& N^{\prime}(1)=Q \lambda E(I)\left\{\left(1-G^{*}(\alpha)\right)(1+\alpha(E(D)+E(R)))+\alpha p G^{*}(\alpha) E(V)\right\} \\
& N^{\prime \prime}(1)=(\lambda E(I))^{2} Q\left\{\alpha\left(1-G^{*}(\alpha)\right)\left[E\left(D^{2}\right)+E\left(R^{2}\right)+2 E(D) E(R)\right]\right. \\
& \left.+2 G^{*^{\prime}}(\alpha)(1+\alpha(E(D)+E(R)))+\alpha p G^{*}(\alpha) E\left(V^{2}\right)-2 p E(V)\left(\alpha G^{*^{\prime}}(\alpha)+G^{*}(\alpha)\right)\right\} \\
& +\lambda E(I(I-1)) Q\left\{\left(1-G^{*}(\alpha)\right)(1+\alpha(E(D)+E(R)))+\alpha p G^{*}(\alpha) E(V)\right\} \\
& +2 \alpha Q \lambda E(I)\left(1-G^{*}(\alpha)\right)(E(D)+E(R)) \\
& D^{\prime}(1)=-\lambda E(I)\left\{\left(1-G^{*}(\alpha)\right)(1+\alpha(E(D)+E(R)))+\alpha p G^{*}(\alpha) E(V)\right\}+\alpha G^{*}(\alpha) \\
& \\
& \quad-\alpha(\lambda E(I))^{2}\left(E\left(D^{2}\right)+E\left(R^{2}\right)+2 E(D) E(R)\right) \\
& \quad-\lambda E(I(I-1))\left\{\left(1-G^{*}(\alpha)\right)\left(1+\alpha(E(D)+E(R))+\alpha p G^{*}(\alpha) E(V)\right\}\right. \\
& -2 \lambda E(I)\left\{1+\alpha G^{*^{\prime}}(\alpha)\{1+\alpha(E(D)+E(R))-\alpha p E(V)\}\right. \\
& D^{\prime \prime}(1)=-\left(\lambda E(I) G^{*^{\prime}}(\alpha)+\alpha\left(1-G^{*}(\alpha)\right)(E(D)+E(R))\right\}
\end{aligned}
$$

The results obtained in equations (71) to (76) agree with the result given by the authors in previous work. (See F. Rehab, Madan, K. C. and Cormac A. L (2010)

\subsection{Case 3. no delay for repairs to start and No extended vacation time}

If there is no delay time, we let $E(D)=0$ and $F^{*}(b)=1$, so we consider there is no extended vacation time we let $r=0$ and $W^{*}(b)=1$. Using this in the main results of this paper, we get 


$$
\begin{aligned}
& S_{q}(z)=\frac{-Q\left(1-G^{*}(a)\right)\left\{b+\alpha z\left(1-H^{*}(b)\right)\right\}-a Q p G^{*}(a)\left\{1-B^{*}(b)\right\}}{a\left(z-G^{*}(a)\left(1-p+p B^{*}(b)\right)\right)-\alpha z\left(1-G^{*}(a)\right) H^{*}(b)} \\
& Q=1-\lambda E(I)\left[\frac{1}{\alpha G^{*}(\alpha)}+\frac{E(R)}{G^{*}(\alpha)}-\frac{1}{\alpha}-E(R)+p E(V)\right] \\
& \left.N^{\prime}(1)=Q \lambda E(I)\left\{\left(1-G^{*}(\alpha)\right)(1+\alpha E(R))\right)+\alpha p G^{*}(\alpha) E(V)\right\} \\
& N^{\prime \prime}(1)=(\lambda E(I))^{2} Q\left\{\alpha\left(1-G^{*}(\alpha)\right) E\left(R^{2}\right)+2 G^{*^{\prime}}(\alpha) E(R)\right. \\
& \left.\quad+\alpha p G^{*}(\alpha) E\left(V^{2}\right)-2 p E(V)\left(\alpha G^{*^{\prime}}(\alpha)+G^{*}(\alpha)\right)\right\} \\
& \quad+\lambda E(I(I-1)) Q\left\{\left(1-G^{*}(\alpha)\right)(1+\alpha E(R))+\alpha p G^{*}(\alpha) E(V)\right\} \\
& \left.+2 \alpha Q \lambda E(I)\left(1-G^{*}(\alpha)\right) E(R)\right) \\
& D^{\prime}(1)=-\lambda E(I)\left\{\left(1-G^{*}(\alpha)\right)(1+\alpha E(R))+\alpha p G^{*}(\alpha) E(V)\right\}+\alpha G^{*}(\alpha) \\
& \\
& D^{\prime \prime}(1)=-(\lambda E(I))^{2} G^{*}(\alpha)\left\{\alpha p E\left(V^{2}\right)-2 p E(V)-\alpha E\left(R^{2}\right)\right\} \\
& -\alpha(\lambda E(I))^{2} E\left(R^{2}\right)-2(\lambda E(I))^{2} G^{*^{\prime}}(\alpha)\{1+\alpha E(R)-\alpha p E(V)\} \\
& -\lambda E(I(I-1))\left\{\left(1-G^{*}(\alpha)\right)(1+\alpha E(R))+\alpha p G^{*}(\alpha) E(V)\right\} \\
& -2 \lambda E(I)\left\{1+\alpha G^{*^{\prime}}(\alpha)+\alpha\left(1-G^{*}(\alpha)\right) E(R)\right\}
\end{aligned}
$$

The results obtained in equations (77) to (82) agree with the result given by Maraghi, Madan, \& Darby-Dowman (2009 a).

\section{Numerical Example}

To numerically illustrate the results obtained in this work we consider that the service times, vacation times, delay times, extended vacation times and repair times are exponentially distributed. The next tables shows the effect of the new contribution of this work, where we study different performance measures under different values of the new parameters $r, \varphi$ and $\theta$ All the values were chosen so that the steady state condition is satisfied.

In Table 2 we choose the following values:

$\mu=7, \beta=6, \gamma=3, \lambda=2, \varphi=5, \theta=7, \alpha=2, E(I)=1$ and $E(I(I-1))=0$,we consider that $r$ takes the values 0 , 0.25 and 0.5 , while $p$ takes the values $0.25,0.5$ and 0.75 .

In Table 3 we consider $\mu=7, \lambda=2, \alpha=2, \theta=7, \gamma=3, r=0.5, p=0.5, E(I)=1$ and $E(I(I-1))=0$, while $\beta$ takes the values 5, 7, 9 and $\varphi$ takes the values 3,5 , and 7 .

In Table 4 we consider $\mu=7, \lambda=2, \alpha=2, \beta=9, \varphi=7, p=0.5, r=0.5, E(I)=1$ and $E(I(I-1))=0$, while $\gamma$ takes the values 3,5 , and 7 and $\theta$ takes the values 4,6 , and 8 .

Table 2 shows that increasing the value of $r$ or $p$ causes increasing the value of utilization factor, the mean queue size and the mean waiting time of the customers while the server idle time decreases.

Table 3 shows that increasing the value of $\beta$ or $\varphi$ causes decreasing the value of utilization factor, the mean queue size and the mean waiting time of the customers while the server idle time increases.

It is clear from Table 4 that as long as we increase the value of $\gamma$ or $\theta$, the server idle time increases while the utilization factor, the mean queue size and the mean waiting time of the customers, all decrease. All the trends shown by the tables are as expected.

\section{References}

Aissani, A. \& Artalejo, J. R. (1998). On the single server retrial queue subject to breakdowns, Queueing Systems, 30 (3-4), pp. 309-321. http://dx.doi.org/10.1023/A:1019125323347

Alfa, A. S. (2003). Vacation models in discrete time, Queueing Systems, 44 (1), pp. 5-30. http://dx.doi.org/10.1023/A:1024 028722553

Altman, E. \& Yechiali, U. (2006). Analysis of customers' impatience in queue with server vacations, Queueing Systems, 52 (4), pp. 261-279. http://dx.doi.org/10.1007/s11134-006-6134-x 
Doshi, B. T. (1986). Queueing systems with vacations - a survey, Queueing Systems, 1, pp. 29-66. http://dx.doi.org/10.1007 /BF01149327

F. Rehab, Madan, K. C. and Cormac, A. L. (2010). An M[X] /G/1 Queue with Bernoulli Schedule, General Vacation Times, Random Breakdowns, General Delay Times and General Repair Times. Applied mathematical Sciences, 6 (1), pp.35-51.

Federgruen, A. \& So, K. C. (1990). Optimal maintenance policies for singleserver queueing systems subject to breakdowns, Operations Research, 38 (2), pp. 330-343. http://dx.doi.org/10.1287/opre.38.2.330

Lee, H. S. \& Srinivasan, M. M. (1989). Control policies for the MX/G/1 queueing system, Management Science, 35 (6), pp. 708-721. http://dx.doi.org/10.1287/mnsc.35.6.708

Li, H. \& Zhu, Y. (1996). Analysis of $M / G / 1$ queues with delayed vacations and exhaustive service discipline, European Journal of Operational Research, 92 (1), pp. 125-134. http://dx.doi.org/10.1016/0377-2217(94)00364-5

Madan, K. C. \& Al-Rawwash, M. (2005). On the $M x / G / 1$ queue with feedback and optional server vacations based on a single vacation policy, Applied Mathematics and Computation, 160, pp. 909-919.

Madan, K. C. (1991). On a $M[x] / M[b] / 1$ queueing system with general vacation times, International Journal of Information and Management Sciences, 2 (1), pp. 51-60.

Madan, K. C. (2000b). On a single server queue with two-stage heterogeneous service and binomial schedule server vacations The Egyptian Statistical Journal, 44 (1), pp. 39-55.

Madan, K. C., Abu-Dayyeh, W. \& Gharaibeh, M. (2003b). Steady state analysis of two Mx/Mab/1 queue models with random breakdowns, International Journal of Information and Management Sciences, 14 (3), pp. 37-51.

Madan, K. C., Al-Rawi, Z. R. \& Al-Nasser, A. D. (2005). On $M x /(G 1 G 2) / 1 / G(B S) / V s$ vacation queue with two types of general heterogeneous service, Journal of Applied Mathematics and Decision Sciences, 2005 (3), pp. $123-135$. http://dx.doi.org/10.1155/JAMDS.2005.123

Maraghi, F. A., Madan, K. C. \& Darby-Dowman, K. (2009a). Batch arrival queueing system with random breakdowns and Bernoulli schedule server vacations having general vacation time distribution, International Journal of Information and Management Sciences, Vol. 20, No. 1, pp. 55-70.

Niu, Z., Shu, T. \& Takahashi, Y. (2003). A vacation queue with setup and closedown times and batch Markovian arrival processes, Performance Evaluation, 54, pp. 225-248. http://dx.doi.org/10.1016/S0166-5316(03)00058-0

Takagi, H. (1990). Time-dependent analysis of $M / G / 1$ vacation models with exhaustive service, Queueing Systems, 6 (1), pp. 369-390. http://dx.doi.org/10.1007/BF02411484

Tian, N. \& Zhang, Z. G. (2002). The discrete-time GI/Geo/1 queue with multiple vacations, Queueing Systems, 40 (3), pp. 283-294. http://dx.doi.org/10.1023/A:1014711529740

Wang, H. \& Li, J. (2008). A repairable $M / G / 1$ Retail queue with Bernoulli vacation and two-phase service, Quality Technology and Quantitative Management, 5 (2), pp. 179-192.

Wang, J., Cao, J. \& Li, Q. (2001). Reliability analysis of the retrial queue with server breakdowns and repairs, Queueing Systems, 38 (4), pp. 363-380. http://dx.doi.org/10.1023/A:1010918926884

Xu, Q., Bao, S., Ma, Z. \& Tian, N. (2007). MX/G/1 queue with multiple vacations, Stochastic Analysis and Applications, 25, pp. 127-140.

Y. Levy, U. Yechilai. (1976). An M/M/s queue with servers vacations, INFOR, 14 (2), pp. 153-163. 
Table 1.

\begin{tabular}{|c|c|}
\hline Notations & Definitions \\
\hline$P_{n}(t, x)$ & $\begin{array}{l}\text { probability that at time } t \text {, there are } n \quad(n \geq 0) \text { customers in the queue excluding the } \\
\text { customer in service and the elapsed service time of this customer is } x\end{array}$ \\
\hline$P_{n}(t)=\int_{0} P_{n}(t, x) d x$ & $\begin{array}{l}\text { probability that there are } n \quad(n \geq 0) \text { customers in the queue excluding the customer in } \\
\text { service irrespective of the value of } x\end{array}$ \\
\hline$V_{n}(t, x)$ & $\begin{array}{l}\text { probability that at time } t \text {, there are } n \quad(n \geq 0) \text { customers in the queue and the server is on } \\
\text { vacation with elapsed vacation time } x\end{array}$ \\
\hline$V_{n}(t)=\int_{0} V_{n}(t, x) d x$ & $\begin{array}{l}\text { probability that at time } t \text {, there are } n \quad(n \geq 0) \text { customers in the queue and the server is on } \\
\text { vacation irrespective of the value of } x\end{array}$ \\
\hline$Q(t)$ & $\begin{array}{l}\text { probability that at time } t \text {, there are no customers in the system and the server is idle but } \\
\text { available in the system. }\end{array}$ \\
\hline$D_{n}(t, x)$ & $\begin{array}{l}\text { probability that at time } t \text {, there are } n(n \geq 0) \text { customers in the queue, and the server is } \\
\text { inactive due to a system breakdown and waiting for repairs to start with elapsed delay } \\
\text { time } x\end{array}$ \\
\hline$D_{n}(t)=\int_{0} D_{n}(t, x) d x$ & $\begin{array}{l}\text { probability that at time } t \text {, there are } n \quad(n \geq 0) \text { customers in the queue and the server is } \\
\text { waiting for repairs to start irrespective of the value of } x\end{array}$ \\
\hline$R_{n}(t, x)$ & $\begin{array}{l}\text { probability that at time } t \text {, there are } n(n \geq 0) \text { customers in the queue, and the server is } \\
\text { under repair with elapsed repair time } x\end{array}$ \\
\hline$R_{n}(t)=\int_{0} R_{n}(t, x) d x$ & $\begin{array}{l}\text { probability that at time } t \text {, there are } n(n \geq 0) \text { customers in the queue and the server under } \\
\text { repairs irrespective of the value of } x\end{array}$ \\
\hline$E_{n}(t, x)$ & $\begin{array}{l}\text { probability that at time } t \text {, there are } n(n \geq 0) \text { customers in the queue, and the server is on } \\
\text { an extended vacation with elapsed extended vacation time } x\end{array}$ \\
\hline$E_{n}(t)=\int_{0} E_{n}(t, x) d x$ & $\begin{array}{l}\text { probability that at time } t \text {, there are } n(n \geq 0) \text { customers in the queue and the server is on } \\
\text { an extended vacation. }\end{array}$ \\
\hline
\end{tabular}

Table 2.

\begin{tabular}{|c|c|c|c|c|c|c|c|}
\hline$r$ & $p$ & $\rho$ & $Q$ & $L_{q}$ & $W_{q}$ & $L$ & $W$ \\
\hline & 0.25 & 0.6412 & 0.3588 & 1.9167 & 0.9584 & 2.5579 & 1.2789 \\
\hline & 0.5 & 0.7245 & 0.2755 & 2.6837 & 1.3419 & 3.4082 & 1.7041 \\
\hline & 0.75 & 0.8078 & 0.1922 & 4.1159 & 2.0579 & 4.9237 & 2.4619 \\
\hline \multirow{3}{*}{0.25} & 0.25 & 0.6662 & 0.3338 & 2.1366 & 1.0683 & 2.8027 & 1.4014 \\
\hline & 0.5 & 0.7745 & 0.2255 & 3.5047 & 1.7523 & 4.2792 & 2.1396 \\
\hline & 0.75 & 0.8828 & 0.1172 & 7.4025 & 3.7013 & 8.2854 & 4.1427 \\
\hline \multirow{3}{*}{0.5} & 0.25 & 0.6912 & 0.3088 & 2.392 & 1.196 & 3.0832 & 1.5416 \\
\hline & 0.5 & 0.8245 & 0.1755 & 4.7934 & 2.3967 & 5.6179 & 2.809 \\
\hline & 0.75 & 0.9578 & 0.0422 & 22.378 & 11.189 & 23.3359 & 11.6679 \\
\hline
\end{tabular}


Table 3.

\begin{tabular}{|l|l|l|l|l|l|l|l|}
\hline$\beta$ & $\varphi$ & $\rho$ & $Q$ & $L_{q}$ & $W_{q}$ & $L$ & $w$ \\
\hline \multirow{4}{*}{5} & 3 & 0.9245 & 0.0755 & 13.2268 & 6.6134 & 14.1513 & 7.0756 \\
\cline { 2 - 8 } & 5 & 0.8578 & 0.1422 & 6.2031 & 3.1015 & 7.0609 & 3.5304 \\
\cline { 2 - 8 } & 7 & 0.8293 & 0.1707 & 4.9356 & 2.4678 & 5.7649 & 2.8824 \\
\hline \multirow{4}{*}{7} & & & & & & & \\
\hline \multirow{4}{*}{9} & 3 & 0.8673 & 0.1327 & 6.967 & 3.4835 & 7.8344 & 3.9172 \\
\cline { 2 - 8 } & 5 & 0.8007 & 0.1993 & 4.0889 & 2.0444 & 4.8895 & 2.4448 \\
\cline { 2 - 8 } & 7 & 0.7721 & 0.2279 & 3.4186 & 1.7093 & 4.1907 & 2.0954 \\
\hline & 3 & 0.8356 & 0.1644 & 5.4041 & 2.702 & 6.2397 & 3.1198 \\
\cline { 2 - 8 } & 5 & 0.7689 & 0.2311 & 3.3906 & 1.6953 & 4.1595 & 2.0798 \\
\cline { 2 - 8 } & 7 & 0.7404 & 0.2596 & 2.8861 & 1.4431 & 3.6265 & 1.8132 \\
\hline
\end{tabular}

Table 4.

\begin{tabular}{|l|l|l|l|l|l|l|l|}
\hline$\theta$ & $\gamma$ & $\rho$ & $Q$ & $L_{q}$ & $W_{q}$ & $L$ & $w$ \\
\hline \multirow{4}{*}{4} & 3 & 0.8016 & 0.1984 & 4.6216 & 2.3108 & 5.4232 & 2.7116 \\
\cline { 2 - 8 } & 5 & 0.7254 & 0.2746 & 2.5479 & 1.2739 & 3.2733 & 1.6366 \\
\cline { 2 - 8 } & 7 & 0.6927 & 0.3073 & 2.0145 & 1.0072 & 2.7072 & 1.3536 \\
\hline \multirow{3}{*}{6} & & & & & & & \\
\hline \multirow{3}{*}{8} & 3 & 0.754 & 0.246 & 3.1879 & 1.594 & 3.9419 & 1.9709 \\
\cline { 2 - 8 } & 5 & 0.6778 & 0.3222 & 1.7991 & 0.8995 & 2.4769 & 1.2384 \\
\hline \multirow{3}{*}{8} & 3 & 0.6451 & 0.3549 & 1.4215 & 0.7107 & 2.0666 & 1.0333 \\
\cline { 2 - 8 } & 5 & 0.7302 & 0.2698 & 2.6829 & 1.3414 & 3.413 & 1.7065 \\
\cline { 2 - 8 } & 7 & 0.654 & 0.346 & 1.5192 & 0.7596 & 2.1731 & 1.0866 \\
\hline
\end{tabular}

